\title{
Contribution of Lower Limb Joint Movement in Adapting to Re-establish Step Length Symmetry During Split-Belt Treadmill Walking
}

\author{
Keisuke Hirata ${ }^{1} \cdot$ Takanori Kokubun $^{2} \cdot$ Taku Miyazawa $^{3} \cdot$ Hikaru Yokoyama $^{4} \cdot$ Keisuke Kubota $^{1} \cdot$ Moeka Sonoo $^{1}$. \\ Hiroki Hanawa ${ }^{1} \cdot$ Naohiko Kanemura ${ }^{1}$ (D)
}

Received: 8 February 2018 / Accepted: 24 October 2018 / Published online: 13 November 2018

(c) The Author(s) 2018

\begin{abstract}
People walking on a split-belt treadmill, where each belt moves at a different speed, adapt step length to re-establish symmetry. However, kinematic changes that occur when re-establishing step length symmetry have not yet been clarified. This study aims to clarify the changing lower limb joint kinematics of each leg when re-establishing step length symmetry. We examined ten young adults who walked on a double-belt treadmill under symmetric and asymmetric conditions of belt velocity using a motion capture system. The results showed that the hip flexion angle at heel strike on one side was not significantly different between the symmetric and asymmetric conditions $(p=0.38)$ thereby demonstrating that the hip flexion angle of the leading leg re-established symmetry at heel strike (HS) of the fast and slow sides. However, the knee extension angle of the leading leg expanded asymmetrically at HS on both sides. The shank of the fast leg possibly accelerated more to move the heel further forward. The results indicated that subjects with limited knee extension function must increase their hip flexion movement of the fast-side limb to accelerate the shank in the swing phase. The present study suggests securing the range of motion and treatment of the knee extension to reduce the spasticity of hamstrings or plantarflexor before split-belt training. Moreover, split-belt training may be undesirable for improving step length symmetry in impaired subjects who are unlikely to improve the function of knee extension movement. The present study contributes toward developing a rehabilitation protocol that improves the gait asymmetry of patients through split-belt training.
\end{abstract}

Keywords Split-belt treadmill $\cdot$ Walking $\cdot$ Adaptation $\cdot$ Step length $\cdot$ Symmetry $\cdot$ Rehabilitation

\section{Introduction}

Naohiko Kanemura

kanemura-naohiko@spu.ac.jp

1 Graduate Course of Health and Social Services, Graduate School of Saitama Prefectural University, 820 Sannomiya, Koshigaya City, Saitama 343-8540, Japan

2 Department of Health and Social Services, Saitama Prefectural University, 820 Sannomiya, Koshigaya City, Saitama 343-8540, Japan

3 Ageo Futatsumiya Clinic, 954-1 Futatsumiya, Ageo City, Saitama 362-0017, Japan

4 Laboratory of Sports Sciences, Department of Life Sciences, Graduate School of Arts and Sciences, The University of Tokyo, 3-8-1 Komaba, Meguro, Tokyo 153-8902, Japan

Able-bodied people show asymmetry in lower limb actions as well as laterality (or leg dominance) during bipedal locomotion [1]. Many studies have investigated this asymmetry. For example, Giakas and Baltzopoulos found that substantial asymmetries characterized time domain variables in the mediolateral component of ground reaction forces [2]. Sadeghi et al. reported local asymmetry between the right and left hips, knees, and ankles in the gait of able-bodied people depending on the functional task to control balance, interlimb coordination, and propulsion functions [3]. However, the cause of such asymmetry in able-bodied people remains unclear [1]. By contrast, gait asymmetry is commonly observed in impaired people during walking, and these are common outcome metrics in clinical rehabilitation. Treadmill and overground walking 
are biomechanically and symmetrically similar in ablebodied [4] and elderly people [5] and patients [6].

Recent studies with split-belt treadmills have demonstrated the adaptability of human bipedal locomotion through spatiotemporal changes in gait symmetry or asymmetry parameters [7, 8]. When people walk on a split-belt treadmill, spatiotemporal parameters such as step length and double support time are initially asymmetric. However, these parameters are eventually adapted to re-establish symmetry despite each belt moving at different speeds. Hemiparetic and Parkinsonian gaits are characterized by asymmetric joint kinematics, kinetics, and step length [9, 10]. Recent studies have demonstrated that split-belt treadmill training improves gait symmetry in post-stroke [11, 12] and Parkinson's disease patients [13]. In post-stroke patients, the improved gait symmetry pattern also transfers to overground walking [14] and carries over day to day [15]. However, studies on reducing the asymmetric step length of post-stroke patients did not obtain common results for patients without subgrouping by baseline asymmetry [11, 12, 15-18]. For example, post-stroke patients, who take a longer paretic step length during baseline, are trained with the paretic leg on the slow belt to induce an effect that leads to greater symmetry. Patients who take a shorter paretic step in baseline are trained with the paretic leg on the fast belt to induce an effect that leads to greater symmetry. If a patient is trained in the wrong direction, he/ she acquires effects that worsen his/her step length asymmetry [11].

One of the solutions to interpret these previous results about patients is to clarify how able-bodied people change the lower limb joint kinematics occurring when re-establishing the step length symmetry. Actually, even for ablebodied people, how the lower limb joint contributes to re-establishing the step length symmetry during split-belt treadmill walking remains unknown. Most previous studies on re-establishing the step length symmetry during split-belt treadmill investigated only the spatiotemporal gait parameters (e.g., step length and stance time). These parameters are not factors, but results of kinematic (e.g., joint angle and joint angular acceleration) and kinetic efforts (e.g., ground reaction force and joint moment). Specifically, at the moment of heel strike (HS), the step length can be calculated from the angle between both legs and the length of both legs [19]. The angle between both legs and the length of both legs are affected by the lower limb joint angles. Therefore, clarifying lower limb joint kinematics during split-belt treadmill walking may help specify the application criteria for subjects and the setting of treadmill condition. If it remains unclear, selecting the belt side according to the state of the patient is difficult. Moreover, interpreting the reason of the phenomenon evoked by split-belt treadmill is difficult. This may hinder the use of split-belt treadmills for rehabilitation.
This study aims to clarify the changing lower limb joint kinematics of each leg when re-establishing step length symmetry. These data will be useful for preselecting patients lacking the joint kinematic evoked to change by the split-belt treadmill. If the split-belt is evoked by the increase of a joint movement, the application criteria are applied to the patient lacking the same joint range. Toward this end, we observed the lower limb joint angles (i.e., hip, knee, and ankle joints) at HS when each belt velocity changes from same to different. This study is expected to demonstrate the effect of split-belt treadmill training and realize the possibility of improving patients' gait asymmetry.

\section{Methods}

\subsection{Subject and Data Collection}

Ten healthy young male adults (age: $21 \pm 1$ years, age range: 20-23 years, height: $1.72 \pm 0.12 \mathrm{~m}$ ) were recruited after checking no previous neural or musculoskeletal disorders by questionnaires. All participants provided written informed consent according to the Declaration of Helsinki before the start of the study. The subjects walked on a double-belt treadmill equipped with force sensors (ITR5018-11, Bertec, US, Fig. 1a), in which each belt's speed was controlled symmetrically or asymmetrically by an independent motor. The participants practiced walking barefoot on a symmetric condition on the treadmill before the experiment. Three-dimensional marker data were collected using a 17-camera (two MX T40-S, eight MX T10, and seven Bonita cameras) Vicon motion capture system (MX Giganet, Nexus2.2.1, Oxford, UK, $100 \mathrm{~Hz}$ ) with the marker setting of the Plug-in-Gait full-body AI model.

\subsection{Experimental Protocol}

For the symmetric condition, both belt speeds were initially $0.9 \mathrm{~m} / \mathrm{s}$ for $1 \mathrm{~min}$, with the last ten strides (five strides each of the slow and fast sides) defined as the baseline period. After confirming that there is no remarkable asymmetry in the comfortable treadmill walking of all subjects, the fast belt was randomly assigned to the right or left side. This is because a previous study reported similar patterns of locomotor adaptation, regardless of which leg walked on the faster belt [8]. The first 10 strides were defined as the earlyadaptation period after increasing the speed of the target side to $1.8 \mathrm{~m} / \mathrm{s}$ with $0.5 \mathrm{~m} / \mathrm{s}^{2}$ acceleration. The subjects were given $3 \mathrm{~min}$ to adjust to the asymmetric condition, with the last 10 strides defined as the late-adaptation period (Fig. 1b). 
A

Vicon motion capture system (17 cameras)

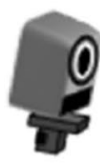

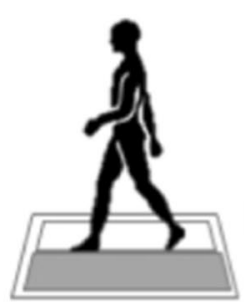

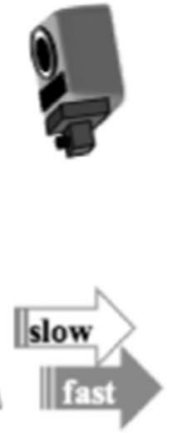

Double belt treadmill
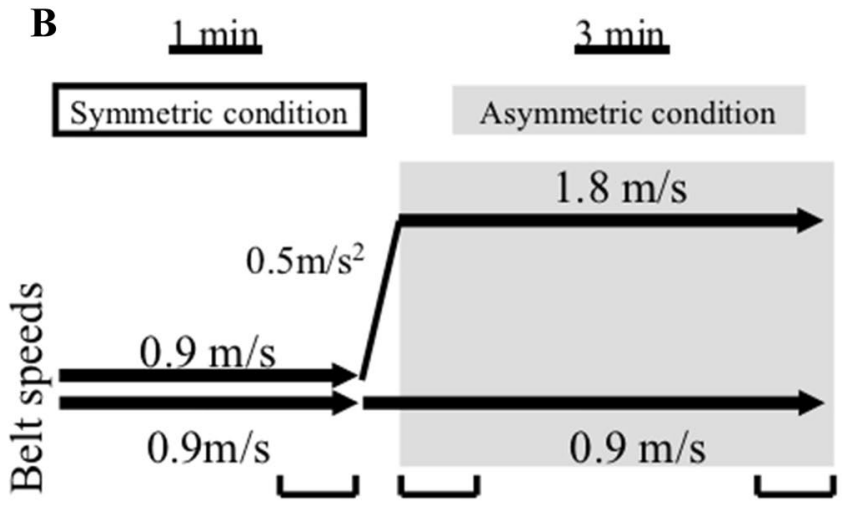

10 strides 10 strides

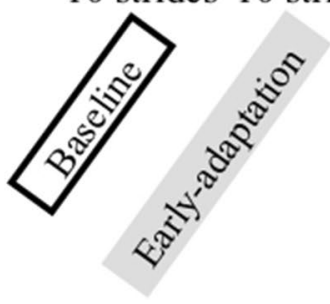

10 strides

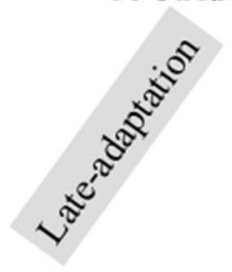

Fig. 1 a Experimental set up. b Gait protocol and collecting periods. For the symmetric condition, both belt speeds were initially $0.9 \mathrm{~m} / \mathrm{s}$ for $1 \mathrm{~min}$, with the last ten strides defined as the baseline period. After increasing the speed of the target side to $1.8 \mathrm{~m} / \mathrm{s}$ with an accel-

\subsection{Data Analysis}

We calculated the following gait parameters. (1) Limb angle at HS (Fig. 2a) and (2) at HS of the opposite limb (Fig. 2b): the angle between a vertical line (against earth vertical) and the vector from the hip to the ankle on sagittal plane. The limb angle was considered positive and negative when the foot was in front of and behind the hip (flexion and extension, respectively). (3) Hip joint flexion angle at HS in the sagittal plane and (4) extension angle at HS of the opposite limb. (5) Knee joint flexion angle at HS in the sagittal plane and (6) flexion angle at HS of the opposite limb. (7) Ankle joint plantar flexion angle at HS in the sagittal plane and (8) plantar flexion angle at HS of the opposite limb. (9) Step length calculated as the anteroposterior distance between the ankle markers of each leg at HS (Fig. 2c, d). (10) Percent stance time: the duration from HS to toe off, expressed as a percentage of the stride time. (11) Percent double support time: the duration from HS to toe off of the opposite limb and vice versa (Fig. 2c, d), expressed as a percentage of the stride time on each limb. Comparative ratios were calculated for all parameters as follows based on a previous study [16].

ratio $=\frac{(\text { data from fast side })-(\text { data from slow side })}{(\text { data from fast side })+(\text { data from slow side })} \times 100(\%)$.

We also calculated the angular accelerations of both the thigh and the shank of the fast and slow side limbs during eration of $0.5 \mathrm{~m} / \mathrm{s}^{2}$, the first 10 strides defined as early-adaptation. The subjects were given 3 min to adjust to the asymmetric condition, with the last ten strides were defined as late-adaptation

the swing phase. We examined whether the forward tilt accelerations of the shank and the thigh have a difference when it comes between the fast and slow side limbs in the late swing phase.

\subsection{Statistics}

The data from the testing periods (i.e., baseline, early-adaptation, and late-adaptation periods) were analyzed using analysis of variance (ANOVA) with repeated measures. When the ANOVA revealed significant differences, the Bonferroni post hoc test was used to compare the testing periods. We further performed paired t-tests to compare the differences between the fast and slow side limbs of the mean accelerations of the shank and the thigh in the late swing phase. The significance level was set to 0.05 . All analyses were performed using MATLAB (MathWorks Inc., USA). The protocol applied in the present study was almost similar to those used in previous studies [8].

\section{Results}

Table 1 lists the parameters. All parameters showed a significant difference between the baseline period and the earlyadaptation period $(p<0.01)$. In the early-adaptation period, all parameters were asymmetric. In the late-adaptation 

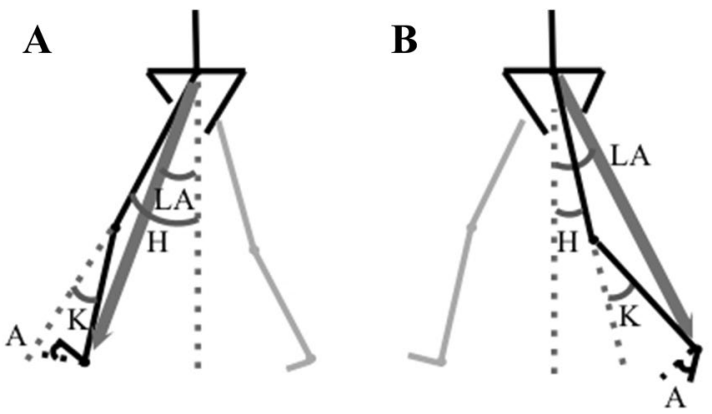

Angles at heel strike

Angles at heel strike on the opposite limb
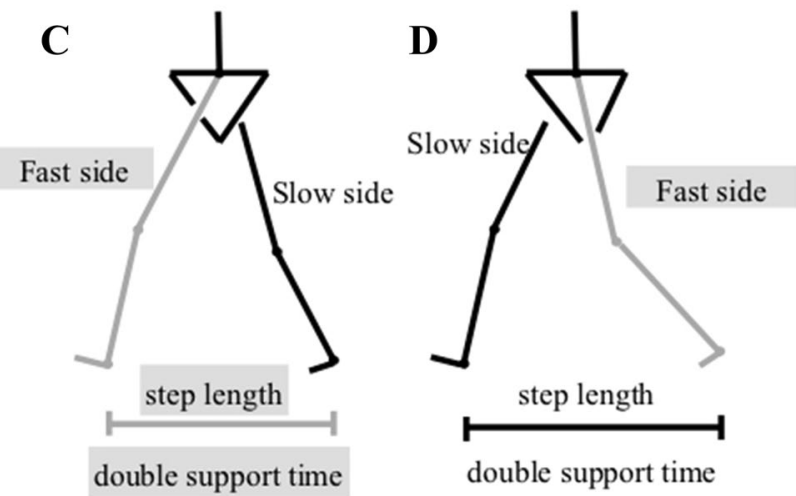

\section{Data from fast side Data from slow side}

Fig. 2 a Definitions of the angles at heel strike. Limb angle (LA), hip flexion angle $(\mathrm{H})$, knee flexion angle $(\mathrm{K})$ and ankle planter flexion angle (A). The limb angle is defined as the angle between a vertical line (against earth vertical) and the vector from the midpoint between the anterior and posterior superior iliac spines to the ankle marker on sagittal plane. b Definitions of the angles at heel strike of the opposite limb. Limb angle (LA), hip extension angle $(\mathrm{H})$, knee flexion angle (K) and ankle planter flexion angle (a). c Definitions of the spatiotemporal parameters. If the left leg is on the fast side belt, the step and the double support time of the fast side are defined when the left leg is the leading leg, and the right leg is the trailing leg. d Similarly, the step length and the double support time of the slow side are defined when the right leg is the leading leg and the left leg is the trailing leg

period, only the step length and hip joint angle at HS were adapted to re-establish symmetry, because no significant difference existed between the baseline and the late-adaptation periods ( $p=1.00$ and 0.38 , respectively), and a significant difference existed between the early- and late-adaptation periods $(p<0.01)$. The other parameters maintained asymmetry in the late-adaptation period because they showed a significant difference between the baseline and late-adaptation periods. Although the percent double support time, the limb angle at HS of the opposite limb and ankle plantar flexion angle at HS on the opposite limb were not adapted to re-establish symmetry, they showed significantly decreased asymmetry from the early- to the late-adaptation period $(p<0.01)$.

We discuss the result for the late-adaptation period (Fig. 3a). At HS of the fast side, the limb angle and knee extension angle of the fast (leading) leg were larger, and the limb angle and knee extension angle of the slow (trailing) leg were smaller. In contrast, at HS on slow side, the limb angle and knee extension angle of the slow (leading) leg were smaller, and the limb angle and knee extension angle of the fast (trailing) leg were larger. In other words, the leading leg was further forward at HS of the fast side than at HS of the slow side. However, the hip flexion angle of the leading leg was the same between HS of the fast and slow sides (Fig. 3b).

Figure 4 shows the mean (over subjects) and standard deviations of the elevation angular acceleration of the thigh and shank during the swing phase. In the early- and lateadaptation periods, the angular accelerations of both the thigh and shank of the fast side limb are visually evident as the change from the baseline period. In particular, in the late swing phase, the forward tilt acceleration of the shank of the fast side limb was significantly larger than that of the slow side limb $(\mathrm{p}<0.05$, slow $=$ mean $-44.90 \pm 4.62$, fast $=$ mean $-39.70 \pm 4.01$ ). In contrast, the acceleration of the thigh of both the fast and slow side limbs was not significantly different $(\mathrm{p}=0.17)$ and approximately 0 (slow $=$ mean $1.96 \pm 1.48$, fast $=$ mean $1.59 \pm 2.06$ )

\section{Discussion}

Previous studies with a split-belt treadmill focused on adaptation and discussed spatiotemporal parameters such as step length, limb angle, and leg support time [8]. However, many studies of patients with step length asymmetry did not obtain common results. For example, the belt condition for re-establishing step length symmetry differed depending on each patient's baseline asymmetry. One solution to interpreting these previous results about patients is the clarification of how to change the lower limb joint kinematics occurring when re-establishing the step length symmetry in ablebodied people. Thus, this study investigates the kinematic effects occurring during split-belt treadmill walking for reestablishing spatial parameter symmetry. For this purpose, we observed the changes in the step length and lower limb joint angles at HS. The results showed that the limb, knee, and ankle angles remained asymmetric in adapted walking on a split-belt treadmill and that the hip flexion angle of the leading leg re-established symmetry. 
Table 1 Means and standard deviations of walking speed of symmetrical index of parameters for baseline, split, and adaptation periods, $p$-values of differences between parameters for the baseline and early- adaptation (EA) periods, the baseline and late-adaptation (LA) periods, and the early-adaptation (EA) and late-adaptation (LA) periods

\begin{tabular}{|c|c|c|c|c|c|c|}
\hline & \multirow[t]{2}{*}{ Baseline } & \multirow[t]{2}{*}{ Early adaption } & \multirow[t]{2}{*}{ Late adaption } & \multicolumn{3}{|l|}{$p$ value } \\
\hline & & & & Baseline versus EA & Baseline versus LA & EA versus LA \\
\hline Percent stance time & $1.00 \pm 0.66$ & $9.24 \pm 2.78$ & $12.43 \pm 2.47$ & $<0.01 *$ & $<0.01 *$ & 1.00 \\
\hline Step length & $2.13 \pm 1.52$ & $12.96 \pm 8.12$ & $2.67 \pm 1.77$ & $<0.01 *$ & 1.00 & $<0.01 *$ \\
\hline Percent double support time & $2.08 \pm 1.48$ & $9.48 \pm 4.42$ & $6.68 \pm 3.62$ & $<0.01 *$ & $<0.01^{*}$ & $<0.01 *$ \\
\hline Limb angle at heel strike & $0.14 \pm 2.79$ & $20.18 \pm 3.73$ & $21.31 \pm 3.31$ & $<0.01 *$ & $<0.01^{*}$ & 0.20 \\
\hline $\begin{array}{l}\text { Limb angle at heel strike on the } \\
\text { opposite limb }\end{array}$ & $0.45 \pm 5.62$ & $18.43 \pm 8.35$ & $15.23 \pm 5.28$ & $<0.01 *$ & $<0.01^{*}$ & $<0.01 *$ \\
\hline Hip flexion angle at heel strike & $-0.74 \pm 18.51$ & $-12.19 \pm 17.75$ & $-4.50 \pm 20.25$ & $<0.01^{*}$ & 0.38 & $<0.01 *$ \\
\hline $\begin{array}{l}\text { Hip flexion angle at heel strike on } \\
\text { the opposite limb }\end{array}$ & $-0.01 \pm 3.26$ & $3.00 \pm 2.90$ & $3.19 \pm 2.78$ & $<0.01 *$ & $<0.01^{*}$ & 0.67 \\
\hline Knee flexion angle at heel strike & $0.14 \pm 31.01$ & $-30.85 \pm 19.59$ & $-55.74 \pm 16.56$ & $<0.01^{*}$ & $<0.01 *$ & 0.02 \\
\hline $\begin{array}{l}\text { Knee flexion angle at heel strike } \\
\text { on the opposite limb }\end{array}$ & $0.17 \pm 13.87$ & $-17.40 \pm 19.41$ & $-25.39 \pm 14.80$ & $<0.01 *$ & $<0.01 *$ & 0.33 \\
\hline $\begin{array}{l}\text { Ankle planar flexion angle at heel } \\
\text { strike }\end{array}$ & $1.42 \pm 11.54$ & $15.82 \pm 7.22$ & $15.26 \pm 6.10$ & $<0.01 *$ & $<0.01 *$ & 0.95 \\
\hline $\begin{array}{l}\text { Ankle planar flexion angle at heel } \\
\text { strike on the opposite limb }\end{array}$ & $-0.34 \pm 3.83$ & $-22.36 \pm 7.71$ & $15.48 \pm 6.85$ & $<0.01 *$ & $<0.01 *$ & $<0.01 *$ \\
\hline
\end{tabular}

*Indicates significant difference

\subsection{Changes in Step Length and Limb Angle}

The step length re-established symmetry in adapted walking on a split-belt treadmill [8]. We also obtained the same results in the present study. The limb angle at HS was the spatial parameter of not re-establishing the symmetry through split-belt walking $[8,20]$. Our result showed that the limb angle at HS was larger of the fast side than of the slow side, as also noted in a previous study [21]. In summary, the present study clarifies that the symmetry of the step length was caused by both the increase in the limb angle of the fast leg and the decrease in the limb angle of the slow leg at HS of the fast side. Furthermore, the present study shows that the symmetry of the step length was caused by both the decrease in the limb angle of the slow leg and the increase in the limb angle of the fast leg at HS of the slow side. As mentioned previously, the step length could be calculated using the limb angle between the leading leg and the trailing leg at HS. Previous studies with a split-belt treadmill revealed the spatial behavior of each leg by analyzing the step length and limb angle through the gait cycle $[8,20]$. They focused on the change in the temporal parameters during split-belt treadmill walking (e.g., limb angle phase or timing of heel strike and toe off) $[20,22,23]$. However, the step length (i.e., each leg's detailed position at HS) remained unclear. The present study clarifies that step length symmetry results from the change in each step position at HS of the fast and slow side limbs. It is important to clarify the kinematic changes occurring in each leg at HS because step length symmetry is re-established by the different kinematic behaviors of each joint of each leg.

\subsection{Kinematical Mechanism for Re-establishing Step Length Symmetry}

Our results showed that the hip flexion angle at HS re-established symmetry. This experiment used belt speeds of 0.9 and $1.8 \mathrm{~m} / \mathrm{s}$. A previous study of symmetric walking with treadmill speeds similar to those in our study reported that the lower limb joint angle changes depending on the walking speed [24]. Our result on each hip flexion angle on HS remained the same (i.e., symmetry) despite each leg moving at different speeds in the split-belt treadmill. Therefore, we suggest that the re-establishment of the hip flexion angle symmetry is a specific phenomenon in split-belt treadmill walking.

At HS of the leading leg, the ankle plantar flexion angle of the fast leg was larger than that of the slow leg (i.e., asymmetry). However, in the limb angle at HS of the leading leg, the influence of the ankle angle may only be a few if the ankle joint plantar flexion angle is large or small because the limb angle is defined by the pelvis and the ankle marker. In contrast, the knee flexion angle of the leading leg directly affects the limb angle of the leading leg. Therefore, our results showed that the increase in the limb angle and the length of the fast leg at HS of the fast side was contributed 
Fig. 3 a Pattern diagrams that express position of lower joints at HS on fast and slow side in the late-adaptation period (top row: position at HS on slow side, bottom row: position at HS on fast side). b Typical time series changes of the lower joint angles of leading leg at HS on fast and slow sides for the whole experimental protocol for a single subject (top row: hip flexion angle, middle row: knee flexion angle, bottom row: ankle plantar flexion angle). Data at HS on fast side are indicated by filled symbols. Data at HS on slow side are indicated by open symbols

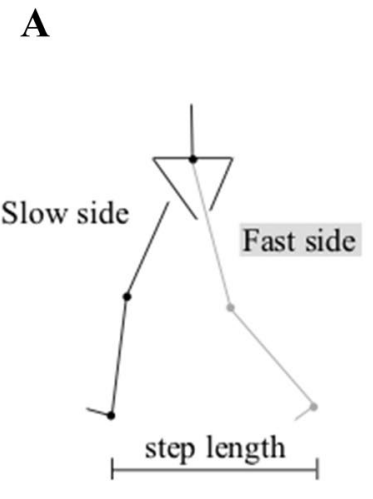

At HS on slow side

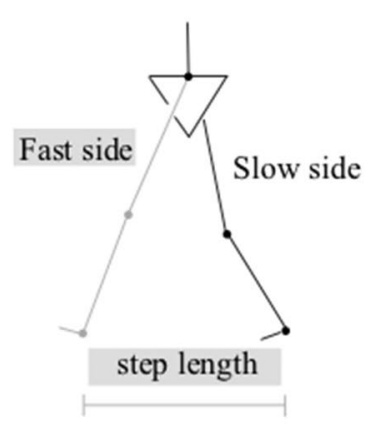

At HS on fast side

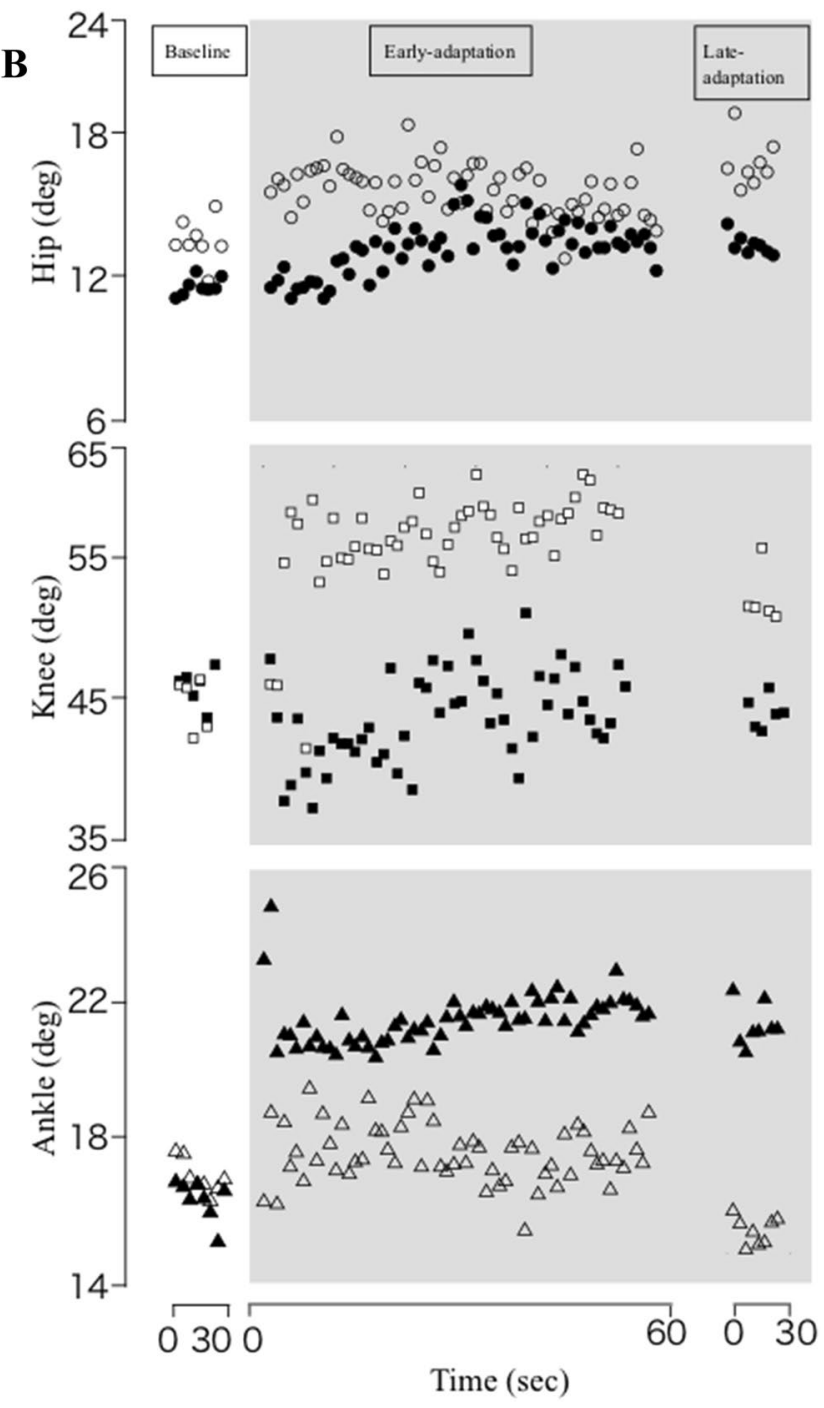

increase their knee extension, they will have to increase the hip flexion movement of the fast side limb.

\subsection{Application to Future Research and Patient Rehabilitation}

Intervention protocols for split-belt treadmill training for post-stroke and Parkinson's disease patients have yet to be established. Previous studies reported that some post-stroke and Parkinson's disease patients could not re-establish step length symmetry even during the late-adaptation period [11, 28]. Our study clarified that not only the step length, but also the hip flexion angle re-established symmetry during splitbelt treadmill walking. Moreover, our study found that to re-establish step length symmetry, the knee extension angle of the fast leg increased at HS. The knee extension function in accelerating the shank forward is another essential factor for re-establishing step length symmetry. For poststroke [29] or Parkinson's disease [30] patients who face 

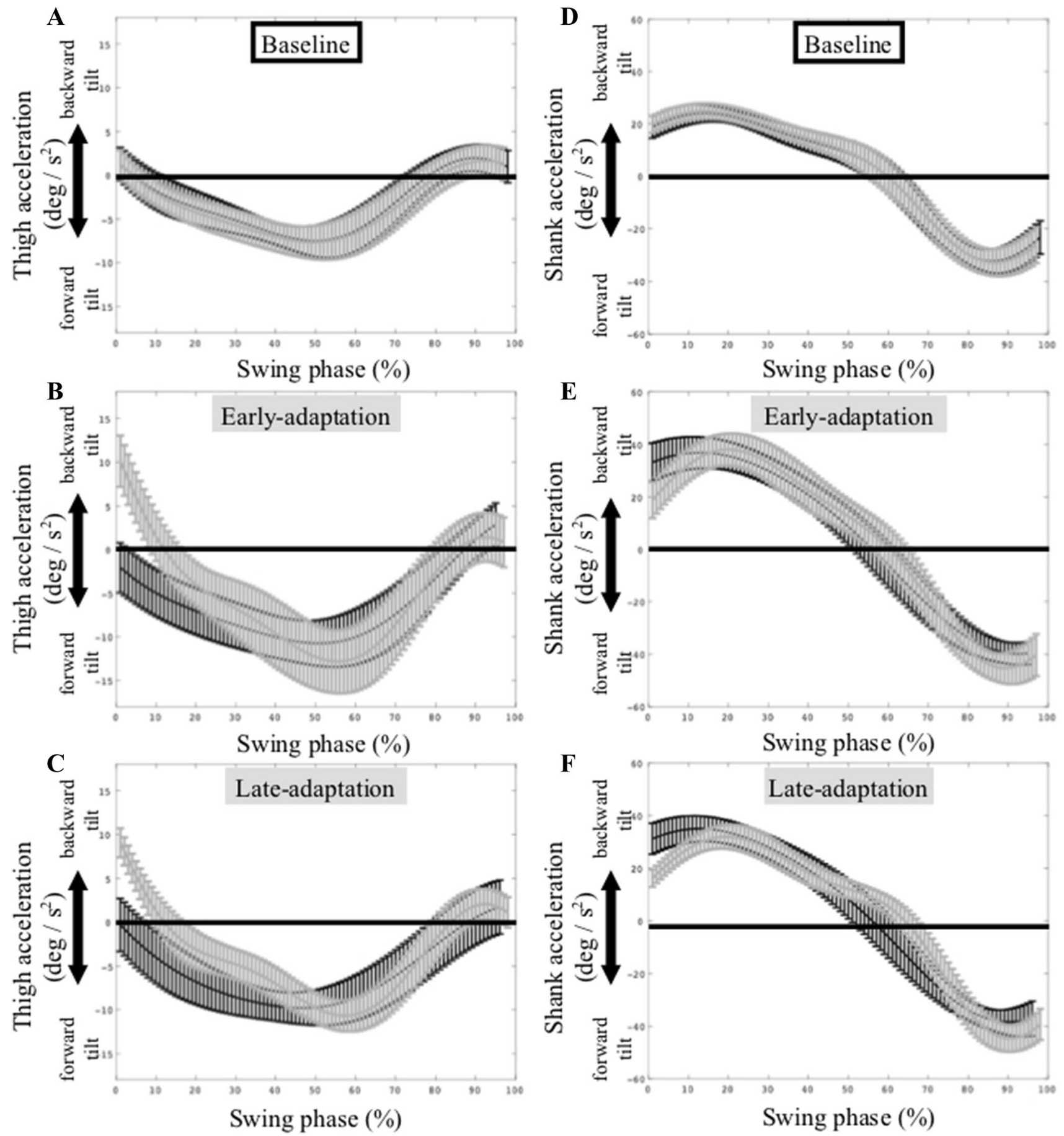

Fig. 4 Mean (over subjects) and standard deviations of the elevation angular acceleration of the thigh and shank during the swing phase. Grey lines indicate data of fast side limb, and black lines indicate data

of slow side limb. Both thigh and shank showed asymmetry during the early-adaptation and late-adaptation periods

spasticity in the hamstrings or joint rigidity in the knee, this result suggests one of the reasons why it is difficult to reestablish step length symmetry during split-belt treadmill walking. It is important to consider that the patient sample includes both individuals who can and cannot increase their

knee extension movement. This may be one of the factors resulting in the difference in the effect of split-belt treadmill among patients in previous studies. We expanded the interpretation of previous research results by subgrouping patients. 
The present study provides important clinical implications such as the necessity of improving the ability to increase knee extension movement before split-belt treadmill training during rehabilitation. If necessary, securing the knee extension range of motion or treatment to reduce the spasticity of the hamstrings or plantarflexor is important. Moreover, it is important to assess kinematical functions for patients to decide the application criteria and settings for the split-belt treadmill. Split-belt treadmill training may not be desirable for improving step length symmetry in impaired subjects who are unlikely to improve the function of knee extension movement. These results should contribute to maximize the split-belt treadmill training effect.

We recruited young male adults, making it unclear whether the finding can be directly generalized to other patient populations. A previous study in split-belt treadmill walking demonstrated that healthy elderly people learned a new walking pattern about the step length similar to younger people [31]. However, this study also mentioned that this result would not normally be considered for the elderly because of the participants' age of early $50 \mathrm{~s}$. Our results should be noted for interpretation because of the subject's asymmetry of walking with age-related weakness. The future study, which will have the same analysis as that in the present study for patients and elderly people, may increase the possibility of clinical application being necessary.

\section{Limitations}

First, our study has a methodological limitation in that only one belt speed condition was considered (2:1 belt speed ratio) and limited results were interpreted. Although most studies $[11,15,20,32,33]$ use a belt speed ratio of $2: 1$, this condition is difficult for application to impaired subjects. For application to patients, the belt speed ratio should be selected based on the ability of the patient.

Second, adaptation may not occur completely if the double support time does not re-establish symmetry in the lateadaptation period. This is expected to be influenced by the shorter walking duration compared with that in the previous study $[8,34]$. In particular, perfect adaptation may occur with a longer asymmetric walking duration. However, we expected these durations to be enough to achieve our main aim of clarifying the process of the change in the lower limb joint movement of each leg from symmetric walking to asymmetric walking.

Third, the change in lower limb joint movement was demonstrated only by analyzing kinematic data. It is important to recognize that the cause of changes in walking patterns can be confirmed by analyzing the muscle activity and kinetic data.

\section{Conclusion}

We clarified the changes in the limb angle and lower limb joint angle when re-establishing step length symmetry using a split-belt treadmill. The results showed that the hip flexion angle of the leading leg re-established symmetry at HS of the fast and slow sides when using a split-belt treadmill. On the other hand, the knee extension angle of the leading leg expanded the asymmetry at HS of the fast and slow sides. The shank of the fast leg possibly accelerated more to move the heel further forward. Therefore, if subjects have limited knee extension function to accelerate the shank in the swing phase, they will be forced to increase the hip flexion movement of the fast side limb. Future studies should aim to establish split-belt treadmill training protocols to improve kinematic asymmetry in the lower limb joints for patients with gait asymmetry by analyzing kinetic data.

\section{Compliance with Ethical Standards}

Conflict of interest All authors declare that they have no conflict of interest.

Open Access This article is distributed under the terms of the Creative Commons Attribution 4.0 International License (http://creativeco mmons.org/licenses/by/4.0/), which permits unrestricted use, distribution, and reproduction in any medium, provided you give appropriate credit to the original author(s) and the source, provide a link to the Creative Commons license, and indicate if changes were made.

\section{References}

1. Sadeghi, H., Allard, P., Prince, F., \& Labelle, H. (2000). Symmetry and limb dominance in able-bodied gait: A review. Gait \& Posture, 12(1), 34-45. https://doi.org/10.1016/S0966 -6362(00)00070-9.

2. Giakas, G., \& Baltzopoulos, V. (1997). Time and frequency domain analysis of ground reaction forces during walking: An investigation of variability and symmetry. Gait \& Posture, 5, 189-197. https://doi.org/10.1016/S0966-6362(96)01083-1.

3. Sadeghi, H. (2003). Local or global asymmetry in gait of people without impairments. Gait \& Posture, 17(3), 197-204. https:// doi.org/10.1016/s0966-6362(02)00089-9.

4. Riley, P. O., Paolini, G., Della Croce, U., Paylo, K. W., \& Kerrigan, D. C. (2007). A kinematic and kinetic comparison of overground and treadmill walking in healthy subjects. Gait Posture, 26(1), 17-24. https://doi.org/10.1016/j.gaitpost.2006.07.003.

5. Parvataneni, K., Ploeg, L., Olney, S. J., \& Brouwer, B. (2009). Kinematic, kinetic and metabolic parameters of treadmill versus overground walking in healthy older adults. Clinical Biomechanics (Bristol, Avon), 24(1), 95-100. https://doi. org/10.1016/j.clinbiomech.2008.07.002.

6. Brouwer, B., Parvataneni, K., \& Olney, S. J. (2009). A comparison of gait biomechanics and metabolic requirements of overground and treadmill walking in people with stroke. Clinical Biomechanics (Bristol, Avon), 24(9), 729-734. https://doi. org/10.1016/j.clinbiomech.2009.07.004. 
7. Dietz, V., Zijkstra, W., \& Duysens, J. (1994). Human neuronal interlimb coordination during split-belt locomotion. Experimental Brain Research, 101, 513-520.

8. Reisman, D. S., Block, H. J., \& Bastian, A. J. (2005). Interlimb coordination during locomotion: What can be adapted and stored? Journal of Neurophysiology, 94(4), 2403-2415. https:// doi.org/10.1152/jn.00089.2005.

9. Fasano, A., Schlenstedt, C., Herzog, J., Plotnik, M., Rose, F. E. M., Volkmann, J., et al. (2016). Split-belt locomotion in Parkinson's disease links asymmetry, dyscoordination and sequence effect. Gait Posture, 48, 6-12. https://doi.org/10.1016/j.gaitp ost.2016.04.020.

10. Olney, S. J., \& Richards, C. (1996). Hemiparetic gait following stroke. Part I: Characteristics. Gait \& Posture, 4, 136-148. https ://doi.org/10.1016/0966-6362(96)01063-6.

11. Reisman, D. S., Wityk, R., Silver, K., \& Bastian, A. J. (2007). Locomotor adaptation on a split-belt treadmill can improve walking symmetry post-stroke. Brain, 130(Pt 7), 1861-1872. https://doi.org/10.1093/brain/awm035.

12. Tyrell, C. M., Helm, E., \& Reisman, D. S. (2015). Locomotor adaptation is influenced by the interaction between perturbation and baseline asymmetry after stroke. Journal of Biomechanics, 48(11), 2849-2857. https://doi.org/10.1016/j.jbiom ech.2015.04.027.

13. Roemmich, R. T., Hack, N., Akbar, U., \& Hass, C. J. (2014). Effects of dopaminergic therapy on locomotor adaptation and adaptive learning in persons with Parkinson's disease. Behavioural Brain Research, 268, 31-39. https://doi.org/10.1016/j. bbr.2014.03.041.

14. Reisman, D. S., Wityk, R., Silver, K., \& Bastian, A. J. (2009). Split-belt treadmill adaptation transfers to overground walking in persons poststroke. Neurorehabil Neural Repair, 23(7), 735-744. https://doi.org/10.1177/1545968309332880.

15. Tyrell, C. M., Helm, E., \& Reisman, D. S. (2014). Learning the spatial features of a locomotor task is slowed after stroke. Journal of Neurophysiology, 112(2), 480-489. https://doi. org/10.1152/jn.00486.2013.

16. Malone, L. A., \& Bastian, A. J. (2014). Spatial and temporal asymmetries in gait predict split-belt adaptation behavior in stroke. Neurorehabilitation Neural Repair, 28(3), 230-240. https ://doi.org/10.1177/1545968313505912.

17. Reisman, D. S., Bastian, A. J., \& Morton, S. M. (2010). Neurophysiologic and rehabilitation insights from the split-belt and other locomotor adaptation paradigms. Physical Therapy, 90, 187-195.

18. Reisman, D. S., McLean, H., Keller, J., Danks, K. A., \& Bastian, A. J. (2013). Repeated split-belt treadmill training improves poststroke step length asymmetry. Neurorehabilitation Neural Repair, 27(5), 460-468. https://doi.org/10.1177/1545968312474118.

19. Kuo, A. D., \& Donelan, J. M. (2010). Dynamic principles of gait and their clinical implications. Physical Therapy, 90(2), 157-174. https://doi.org/10.2522/ptj.20090125.

20. Malone, L. A., Vasudevan, E. V., \& Bastian, A. J. (2011). Motor adaptation training for faster relearning. Journal of Neuroscience, 31(42), 15136-15143. https://doi.org/10.1523/JNEUR OSCI.1367-11.2011.

21. Malone, L. A., Bastian, A. J., \& Torres-Oviedo, G. (2012). How does the motor system correct for errors in time and space during locomotor adaptation? Journal of Neurophysiology, 108(2), 672683. https://doi.org/10.1152/jn.00391.2011.

22. Choi, J. T., \& Bastian, A. J. (2007). Adaptation reveals independent control networks for human walking. Nature Neuroscience, 10(8), 1055-1062. https://doi.org/10.1038/nn1930.

23. Malone, L. A., \& Bastian, A. J. (2010). Thinking about walking: effects of conscious correction versus distraction on locomotor adaptation. Journal of Neurophysiology, 103(4), 1954-1962. https ://doi.org/10.1152/jn.00832.2009.

24. Hutin, E., Pradon, D., Barbier, F., Bussel, B., Gracies, J. M., \& Roche, N. (2012). Walking velocity and lower limb coordination in hemiparesis. Gait Posture, 36(2), 205-211. https://doi. org/10.1016/j.gaitpost.2012.02.016.

25. Neptune, R. R., Sasaki, K., \& Kautz, S. A. (2008). The effect of walking speed on muscle function and mechanical energetics. Gait Posture, 28(1), 135-143. https://doi.org/10.1016/j.gaitp ost.2007.11.004.

26. Neptune, R. R., Clark, D. J., \& Kautz, S. A. (2009). Modular control of human walking: A simulation study. Journal of Biomechanics, 42(9), 1282-1287. https://doi.org/10.1016/j.jbiom ech.2009.03.009.

27. Neptune, R. R., \& McGowan, C. P. (2011). Muscle contributions to whole-body sagittal plane angular momentum during walking. Journal of Biomechanics, 44(1), 6-12. https://doi.org/10.1016/j. jbiomech.2010.08.015.

28. Roemmich, R. T., Nocera, J. R., Stegemoller, E. L., Hassan, A., Okun, M. S., \& Hass, C. J. (2014). Locomotor adaptation and locomotor adaptive learning in Parkinson's disease and normal aging. Clinical Neurophysiology, 125(2), 313-319. https://doi. org/10.1016/j.clinph.2013.07.003.

29. Routson, R. L., Clark, D. J., Bowden, M. G., Kautz, S. A., \& Neptune, R. R. (2013). The influence of locomotor rehabilitation on module quality and post-stroke hemiparetic walking performance. Gait Posture, 38(3), 511-517. https://doi.org/10.1016/j. gaitpost.2013.01.020.

30. Svehlik, M., Zwick, E. B., Steinwender, G., Linhart, W. E., Schwingenschuh, P., Katschnig, P., et al. (2009). Gait analysis in patients with Parkinson's disease off dopaminergic therapy. Archives of Physical Medicine and Rehabilitation, 90(11), 1880 1886. https://doi.org/10.1016/j.apmr.2009.06.017.

31. Malone, L. A., \& Bastian, A. J. (2016). Age-related forgetting in locomotor adaptation. Neurobiology of Learning and Memory, 128, 1-6. https://doi.org/10.1016/j.nlm.2015.11.003.

32. Mawase, F., Haizler, T., Bar-Haim, S., \& Karniel, A. (2013). Kinetic adaptation during locomotion on a split-belt treadmill. Journal of Neurophysiology, 109(8), 2216-2227. https://doi. org/10.1152/jn.00938.2012.

33. Ogawa, T., Kawashima, N., Ogata, T., \& Nakazawa, K. (2014). Predictive control of ankle stiffness at heel contact is a key element of locomotor adaptation during split-belt treadmill walking in humans. Journal of Neurophysiology, 111(4), 722-732. https ://doi.org/10.1152/jn.00497.2012.

34. Yokoyama, H., Sato, K., Ogawa, T., Yamamoto, S. I., Nakazawa, K., \& Kawashima, N. (2018). Characteristics of the gait adaptation process due to split-belt treadmill walking under a wide range of right-left speed ratios in humans. PLOS ONE, 13(4), e0194875. https://doi.org/10.1371/journal.pone.0194875. 